\begin{abstract}
High Plasma Exposure of Statins Associated With Increased Risk of

OPEN ACCESS

Edited by:

Gerfried Karl Hans Nell,

NPC Nell Pharma Connect Ltd,

Austria

Reviewed by:

Robert L. Lins,

Independent Researcher, Belgium

Domenico Criscuolo,

Genovax S.r.l., Italy

Kurt Neumann,

E-I-S Executive Information Service

Kft, Hungary

*Correspondence:

Qingshan Geng

gengqsh@163.net

Min Huang

huangmin@mail.sysu.edu.cn

Shilong Zhong

zhongs/@hotmail.com

${ }^{\dagger}$ These authors have contributed equally to this work and are considered co-first authors.

Specialty section: This article was submitted to Pharmaceutical Medicine and

Outcomes Research,

a section of the journal

Frontiers in Pharmacology

Received: 08 December 2017

Accepted: 11 April 2018

Published: 30 April 2018

Citation:

Cai L, Bai X, Lei H, Wu H, LiU Y, Zhu Q, Zhang S, Liu Y, Lin Q, Chen J, Zhang $B, H e$ G, Geng $Q$, Huang $M$ and Zhong S (2018) High Plasma Exposure of Statins Associated With Increased Risk of Contrast-Induced Acute Kidney Injury in Chinese Patients With Coronary Artery

\section{Contrast-Induced Acute Kidney Injury in Chinese Patients With Coronary Artery Disease}

\author{
Liyun Cai ${ }^{1,2 \dagger}$, Xue Bai ${ }^{1,2,3 \dagger}$, Heping Lei ${ }^{1,2 \dagger}$, Hong Wu ${ }^{4 \dagger}$, Yong Liu ${ }^{1,2}$, Qian Zhu ${ }^{1,2}$, \\ Shanshan Zhang ${ }^{1,2}$, Yibin Liu ${ }^{1,2}$, Qiuxiong Lin ${ }^{1,2}$, Jiyan Chen ${ }^{1,2}$, Bin Zhang ${ }^{1,2}$, \\ Guodong He ${ }^{1,2}$, Qingshan Geng ${ }^{1,2 *}$, Min Huang ${ }^{3 *}$ and Shilong Zhong ${ }^{1,2 *}$ \\ ${ }^{1}$ Guangdong Provincial Key Laboratory of Coronary Heart Disease Prevention, Guangdong Cardiovascular Institute, \\ Guangdong General Hospital, Guangdong Academy of Medical Sciences, Guangzhou, China, ${ }^{2}$ School of Medicine, South \\ China University of Technology, Guangzhou, China, ${ }^{3}$ Laboratory of Drug Metabolism and Pharmacokinetics, School of \\ Pharmaceutical Sciences, Sun Yat-sen University, Guangzhou, China, ${ }^{4}$ Sun Yat-sen Memorial Hospital, Sun Yat-sen \\ University, Guangzhou, China
}

The role of statins in reducing the incidence of contrast-induced acute kidney injury (Cl-AKI) remains controversial. We sought to evaluate the association between $\mathrm{Cl}-\mathrm{AKI}$ and high plasma exposure of statins in coronary artery disease (CAD) patients undergoing coronary angiography (CAG). This association was first evaluated in 1,219 patients with CAD receiving atorvastatin (AT) therapy and validated in 635 patients receiving rosuvastatin (RST) therapy. The plasma concentrations of statins were quantified using validated UPLC-MS/MS methods and Cl-AKI incidence was assessed during the first 48 h postoperatively. Among all participants $(n=1,854)$, AKI occurred in 57 of 1219 $(4.7 \%)$ in the AT cohort and 30 of 635 (4.7\%) in the RST cohort. High plasma AT-all exposure was associated with increased risk of Cl-AKI (odds ratio [OR]: 2.265; 95\% confidence interval [Cl]: 1.609-3.187; $p<0.0001)$. Plasma AT-all concentration in the $\mathrm{Cl}$-AKI group $(22.40 \pm 24.63 \mathrm{ng} / \mathrm{mL})$ was 2.6 -fold higher than that in the control group $(8.60 \pm 9.65 \mathrm{ng} / \mathrm{mL})$. High plasma RST exposure also significantly increased the risk of Cl-AKI (OR: 2.281; 95\% Cl: 1.441-3.612; $p=0.0004)$. We further divided patients into two subgroups for each statin according to baseline renal function, and association between high plasma statin exposure and $\mathrm{Cl}-\mathrm{AKI}$ still remained highly significant in both subgroups. This study suggests for the first time that high plasma exposure of statins may significantly increase the risk of $\mathrm{Cl}$-AKI. Statins should be used with greater caution in CAD patients undergoing CAG to reduce the occurrence of $\mathrm{Cl}-\mathrm{AKI}$.
\end{abstract} Disease. Front. Pharmacol. 9:427. doi: 10.3389/fphar.2018.00427
Keywords: contrast-induced acute kidney injury, coronary angiography, atorvastatin, rosuvastatin, plasma exposure 


\section{INTRODUCTION}

Contrast-induced acute kidney injury (CI-AKI) is a known complication of intravascular administration of contrast media used in coronary angiography (CAG) and percutaneous coronary interventions (PCI) (Chalikias et al., 2016); and is associated with increased mortality, morbidity, healthcare expenditure, and prolonged hospital stay (Nash et al., 2002; Prasad et al., 2016). CI-AKI has become the third leading cause of iatrogenic renal failure in the United States (Prasad et al., 2016). Previous report indicated that even mild postoperative AKI is independently associated with an almost 5-fold increase in in-hospital death (Birnie et al., 2014). Clinically, the incidence of CI-AKI is greater in patients with cardiovascular diseases or pre-existing renal insufficiency (Goldenberg and Matetzky, 2005; Itoh et al., 2005; Ledneva et al., 2009). The reported incidence ranges from 2 to 50\% (Aurelio and Durante, 2014).

Hydroxy-methylglutaryl-coenzyme A (HMG-CoA) reductase inhibitors (statins) are potent inhibitors of cholesterol biosynthesis and exert beneficial effects in the primary and secondary prevention of coronary artery disease (CAD). The prophylactic benefit of statins in reducing the incidence of CI-AKI has been investigated in several observational (Khanal et al., 2005; Patti et al., 2008; Lev et al., 2009) and randomized studies (Patti et al., 2011); however, other studies have reported inconsistent and conflicting results (Argalious et al., 2010; Mithani et al., 2011; Billings et al., 2016; Park et al., 2016). Therefore, whether preoperative statin therapy has a preventive, neutral, or detrimental role on AKI remains unclear and hotly debated. To the best of our knowledge, no studies have evaluated the relationship between high plasma exposure of statins and the risk of CI-AKI.

Therefore, the objective of the current study was to systematically investigate the effects of high plasma exposure of widely prescribed statins (atorvastatin [AT] and rosuvastatin [RST]) and their metabolites on the incidence of CI-AKI in patients with CAD undergoing CAG.

\section{METHODS}

\section{Ethics Statement}

The present study was approved by the Medical Ethical Review Committee of Guangdong General Hospital and conducted according to the Declaration of Helsinki. All participants gave written informed consent in accordance with the Declaration of Helsinki.

\section{Study Design and Patients}

We conducted a prospective two-stage study to evaluate the effects of two statins on CI-AKI separately. In stage I (test set), 1,219 patients taking AT were recruited, including 1,023 patients without chronic kidney disease (CKD) and 196 patients with CKD. In stage II (validation set), 635 patients taking RST were enrolled for further validation; 531 of these were without CKD, whereas 104 were with CKD.

All patients were sequentially recruited in Guangdong General Hospital from January 2010 to December 2013 according to the same inclusion and exclusion criteria. Baseline information, including demographics, medical history, biochemical measurements, and medication was obtained from the hospital information database. Patients who underwent CAG and were diagnosed with CAD were included in the study.

The exclusion criteria included the followings: (1) age $<18$ years or age $>80$ years; (2) renal transplantation or dialysis; (3) liver insufficiency (defined as serum transaminase concentrations $>3$ times the upper limit of normal [120 $\mathrm{U} / \mathrm{L}$ ], or a diagnosis of cirrhosis); (4) being pregnant or lactating; (5) advanced cancer or haemodialysis; (6) the concentrations of statins or their metabolites were lower than limit of detection (3:1 noise).

\section{Coronary Angiography Procedure}

CAG is performed to define the extent and severity of CAD in patients with suspected symptoms whose clinical characteristics and results of non-invasive testing indicate a high likelihood of $\mathrm{CAD}$ and who are amenable to, and candidates for, coronary revascularization (Fihn et al., 2014). Information derived from the CAG procedure will be took into consideration in patient management, and the risks and benefits of the procedure have been carefully considered and understood by the patients.

We used the Synergy between PCI with TAXUS and Cardiac Surgery (SYNTAX) score, an angiographic scoring system to determine the complexity, severity, and atherosclerotic burden of CAD (Sianos et al., 2005; Ikeda et al., 2012). SYNTAX score has been shown to independently predict MACE and longterm prognosis risks in stable CAD patients who underwent revascularization (Serruys et al., 2009; Mohr et al., 2013).

Images of coronary angiograms were obtained with Syngo Dynamics cardiovascular imaging software (Siemens Medical Solutions USA, Inc., Malvern, Pennsylvania). The SYNTAX score was calculated for each patient using a computer program consisting of sequential and interactive self-guided questions according to the SYNTAX score calculator version 2.11. The SYNTAX score reflects a comprehensive anatomical assessment, and a low SYNTAX score was defined as $\leq 22$, an intermediate score as 23 to 32 , and a high score as $\geq 33$.

\section{Clinical Endpoint}

The study endpoint was the diagnosis of postoperative CI-AKI. According to the Acute Kidney Injury Network criteria (Mehta et al., 2007), CI-AKI was diagnosed if a patient had an absolute increase in serum creatinine $(\mathrm{sCr})$ concentration $\geq 0.3 \mathrm{mg} / \mathrm{dL}$ $(26.4 \mu \mathrm{mol} / \mathrm{L})$ from baseline or a relative increase $\geq 50 \%$ (1.5-fold from baseline) in sCr concentration for more than $6 \mathrm{~h}$ within $48 \mathrm{~h}$ after surgery.

CKD was defined as an estimated glomerular filtration rate (eGFR) $<60 \mathrm{~mL} / \mathrm{min}$ per $1.73 \mathrm{~m}^{2}$ using the Modification of Diet in Renal Disease equation (National Kidney Foundation, 2002). SCr levels were measured upon admission and within $48 \mathrm{~h}$ after surgery. Alanine aminotransferase (ALT), aspartate aminotransferase (AST), cholesterol, creatine kinase (CK), creatine kinase $\mathrm{MB}$ (CKMB), and other standard clinical parameters were measured in the morning before the procedure. 


\section{Plasma Sample Preparation}

Each eligible patients had been taking the same dose of AT or RST for at least 7 days prior to blood sampling. Statin dosage was prescribed by physician in accordance with patients' condition. Blood samples were obtained at $10-12 \mathrm{~h}$ post-dose in the morning before the CAG and collected in EDTA-coated tubes. Plasma was separated within $2 \mathrm{~h}$ by centrifugation at 3,000 rpm for $10 \mathrm{~min}$ at $4^{\circ} \mathrm{C}$ and then stored at $-80^{\circ} \mathrm{C}$ until analysis.

\section{Quantification of Plasma Concentrations of Statins and Their Metabolites}

A reliable assay of ultra-performance liquid chromatography coupled with tandem mass spectrometry (UPLC-MS/MS) was developed and validated for the quantification of AT, its five metabolites, and internal standard (IS) carbamazepine in human plasma as described previously (Cai et al., 2017).

A sensitive UPLC-MS/MS assay was also developed and validated for the simultaneous quantification of RST, rosuvastatin lactone (RSTL), and N-desmethyl rosuvastatin (DM-RST) in human plasma. All the three analytes and the corresponding IS (carbamazepine) were extracted from $200 \mu \mathrm{L}$ buffered human plasma (adding $100 \mu \mathrm{L}$ ammonium acetate of $\mathrm{pH}=4.0$ to 100 $\mu \mathrm{L}$ human plasma) by liquid-liquid extraction with ethyl acetate and then separated on an ACQUTY UPLC HSS T3 column (3.0 $\times 100 \mathrm{~mm}, 1.8 \mu \mathrm{m})$. The elution was performed at a rate of 0.3 $\mathrm{mL} / \mathrm{min}$ using a mobile phase containing acetonitrile and $0.05 \%$ formic acid in water over a linear gradient of 30-85\% acetonitrile. Mass detection was performed on a Waters Xevo TQ-S triplequadrupole mass spectrometer in positive electrospray ionization mode. The responses of RST, RSTL, and DM-RST were optimized at the $\mathrm{m} / \mathrm{z} 482.1 \rightarrow 258.1, \mathrm{~m} / \mathrm{z} 464.1 \rightarrow 270.1, \mathrm{~m} / \mathrm{z} 468.0 \rightarrow 258.0$, respectively.

\section{Statistical Analysis}

The demographic and clinical characteristics were summarized using counts (percentages) for the categorical variables and mean (standard deviation, $S D$ ) for the continuous variables. As the ranges of the concentrations of statins and metabolites were skewed, logarithmic transformation was performed prior to analysis. AT-all is calculated amount of plasma AT concentration and equivalent concentrations of its two pharmacologically equipotent metabolites; this value represents overall therapeutic efficacy (Lennernas, 2003). Given that approximate 90\% of plasma pharmacologically activity is accounted for RST, we only applied plasma RST concentration to represent the overall therapeutic efficacy (White, 2002).

Linear regression analysis was applied to evaluate the effects of the baseline demographic and clinical characteristics on the plasma concentrations of statins and metabolites. A univariate logistic regression analysis was conducted to evaluate the effects of plasma concentrations, baseline demographic, and clinical characteristics on the risk to CI-AKI and to calculate odds ratio (OR) and 95\% confidence interval (CI). Variables with $p<0.05$ were entered into the multivariate model, and only variables with $p<0.05$ were retained in the model. $P<0.05$ was considered statistically significant. Data analysis was performed using SAS 9.4 (SAS Inst, Cary, NC, USA).

\section{Predictive Diagnostic Power of Variables for Cl-AKI}

In the study, the Daim package in R (version 3.2.3, http://www. R-project.org/) was used to construct the classification models. For each predictor variable, the true positive rate and false positive rate as a predictor of CI-AKI was evaluated by the receiver operating characteristic (ROC) curves using the area under the curve (AUC) as a measure of diagnostic effectiveness (Zweig and Campbell, 1993). First, every independent variable associated with CI-AKI were selected to construct the classifier for estimating the diagnostic effectiveness of a single predictor. Then, all significant variables were combined as a classifier for estimating the diagnostic effectiveness of variable combinations. The optimal cutoffs were calculated by selecting the data point that maximized the true positive rate and minimized the false positive rate.

\section{RESULTS}

\section{Patient Characteristics and Their Effects on Plasma Statins and Metabolites Exposure}

An overview of the enrolment of the patients is presented in Figure 1. In stage I, plasma concentrations of AT and its metabolites widely varied, which is consistent with published data (DeGorter et al., 2013). The concentrations of five metabolites were highly correlated with AT concentration (all $r>0.5$, $p<0.0001)$. Among 1,219 patients with AT therapy, $21(1.72 \%)$ were taking $10 \mathrm{mg}$ AT, 1058 (86.79\%) were $20 \mathrm{mg}$ AT, and 140 (11.48\%) were $40 \mathrm{mg}$ AT compliance with prescription, respectively. Patients' baseline characteristics and their impacts on the AT concentration are summarized in Table $\mathbf{1 .}$

Univariate linear regression analysis showed that plasma ATall concentration was affected by age, dosage, SYNTAX score, level of ALT, AST, Scr, eGFR, and other clinical parameters. Among these variables, increasing age (estimate $=0.0140$, $p<0.0001$ ), higher dosage (estimate $=0.0173, p=0.0002$ ) higher SYNTAX score (estimate $=0.0059, p=0.0161$ ), higher level of ALT (estimate $=0.0059, p=0.0012$ ), and Scr (estimate $=0.0012, p=0.0008$ ) were independently associated with a higher plasma AT-all concentration (Table 1).

In stage II, of the patients with RST therapy, 11 (1.74\%) were taking $5 \mathrm{mg}$ RST, 549 (86.73\%) were $10 \mathrm{mg}$ RST, 67 (10.58\%) were $20 \mathrm{mg}$ RST and 6 (0.95\%) were $40 \mathrm{mg}$ RST. Multiple linear regression analysis showed that plasma RST concentration was lower in patients with lower level of AST (estimate $=-0.0059$, $p=0.0286$ ) and using angiotensin converting enzyme inhibitors (estimate $=-0.3584, p=0.0320)$ (Table 2).

\section{Effects of Baseline Characteristics and Plasma Exposure of AT and Metabolites on CI-AKI}

In patients receiving AT therapy in stage I, the clinical endpoint CI-AKI within $48 \mathrm{~h}$ after the surgery occurred in $57(4.7 \%)$ patients. Univariate logistic analysis showed that higher plasma exposure of AT-all, AT and its five metabolites, SYNTAX core, 


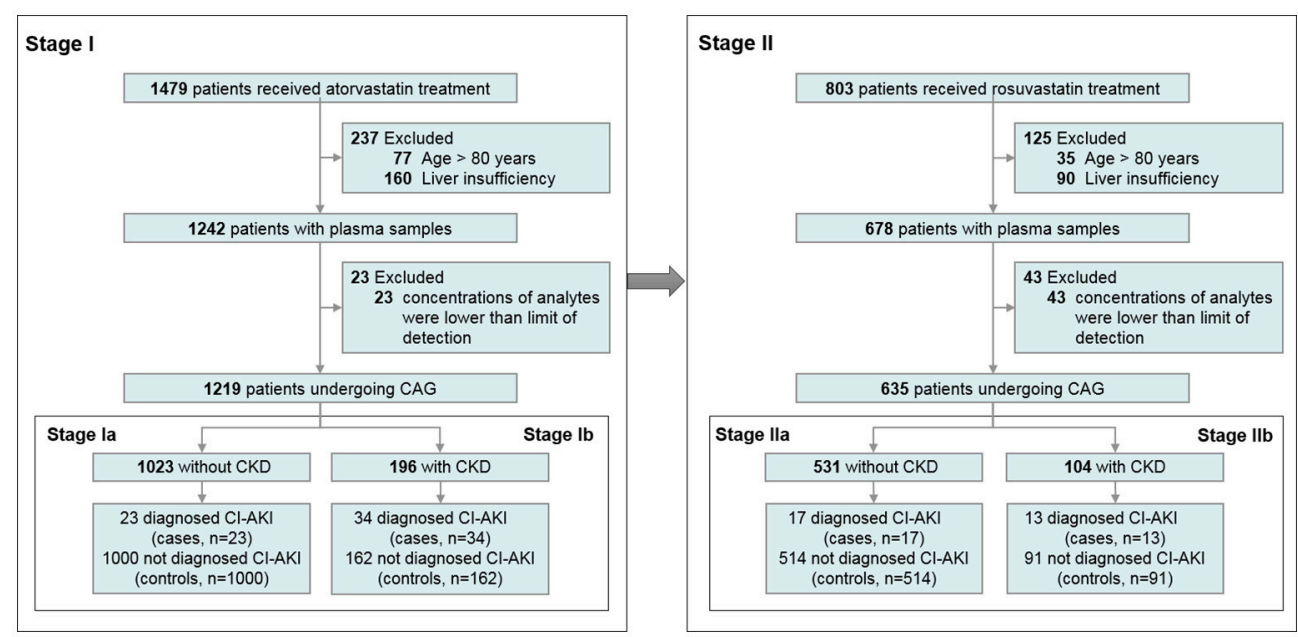

FIGURE 1 | Flow chart of the enrolment of the participants. CAG, coronary angiography; Cl-AKl, contrast-induced acute kidney injury; CKD, chronic kidney disease.

and other factors were associated with a higher risk of CIAKI (Table 3). Multivariate analysis revealed that high plasma AT-all exposure (OR: 2.265; 95\% CI: 1.609-3.187; $p<0.0001$ ), diabetes (OR: 1.953; 95\% CI: 1.030-3.704; $p=0.0403$ ), high level of AST (OR: 1.009; 95\% CI: 1.004-1.015; $p=0.0013$ ), Scr (OR: 1.003; 95\% CI: 1.001-1.006; $p=0.0118$ ), eGFR (OR: 0.977; 95\% CI: $0.964-0.991 ; p=0.0017)$, and use of proton pump inhibitors (PPIs) (OR: 3.979; 95\% CI: 1.828-8.659; $p=0.0005$ ) were independent risk factors for CI-AKI (Table 3). Plasma ATall concentration in patients with CI-AKI $(22.40 \pm 24.63 \mathrm{ng} / \mathrm{mL})$ was 2.6-fold higher than that in controls $(8.60 \pm 9.65 \mathrm{ng} / \mathrm{mL})$

(Figure 2C).

Considering the impact of baseline renal function on CIAKI, we further divided patients into two subgroups, which were patients without CKD and with CKD. In patients without CKD in stage Ia, CI-AKI occurred in 23 (2.2\%) patients. In multivariate logistic model, only plasma AT-all exposure (OR: 2.381 95\% CI: $1.459-3.884 ; p=0.0005$ ), male (OR: 0.327; 95\% CI: 0.133-0.805; $p=0.0150$ ), CK level (OR: 1.001; 95\% CI: $1.000-1.001 ; p=0.0030$ ), and use of PPIs (OR: 10.128; 95\% CI: 2.330-44.028; $p=0.0020)$ were retained in the model implying that they were independent risk factors for CI-AKI (Table S1). Plasma AT-all concentration in patients with CI-AKI $(19.88 \pm 23.69 \mathrm{ng} / \mathrm{mL})$ was 2.5 fold higher than that in controls $(8.08 \pm 8.57 \mathrm{ng} / \mathrm{mL})$ (Figure 2A).

In patients with CKD in stage Ib, CI-AKI occurred in 34 (17.3\%) patients, which is much higher than that in stage Ia. The association between high plasma AT-all exposure and CIAKI remained highly significant. Multivariate logistic regression analysis revealed that high plasma AT-all exposure (OR: 5.377 95\% CI: 2.403-12.032; $p<0.0001$ ) was still an independent risk factors for CI-AKI (Table S2). Plasma AT-all concentration in patients with CI-AKI $(24.10 \pm 25.45 \mathrm{ng} / \mathrm{mL})$ was 2.0 -fold higher than that in controls $(11.82 \pm 14.29 \mathrm{ng} / \mathrm{mL})$ (Figure 2B).

\section{Effects of Baseline Characteristics and Plasma Exposure of RST and Metabolites on CI-AKI}

To further confirm the predictive value of plasma statin concentration for CI-AKI, we assessed the association in patients who received RST treatment in stage II, and the association between high plasma statin exposure and CI-AKI remained highly significant. CI-AKI occurred in 30 (4.7\%) patients who received RST treatment. Multivariate logistic regression analysis revealed that patients in the CI-AKI group had higher plasma RST exposure (OR: 2.281; 95\% CI: 1.441-3.612; $p=0.0004$ ), higher dosage (OR: 1.088; 95\% CI: 1.015-1.167; $p=0.0175$ ), and were with more diabetes (OR: 2.680 ; 95\% CI: 1.153-6.230; $p=0.0220$ ), heart failure (OR: 7.904; 95\% CI: 3.032-20.606; $p<0.0001$ ) (Table 4). RST plasma concentration in the CI-AKI group $(8.28 \pm 5.49 \mathrm{ng} / \mathrm{mL})$ was 2.7 -fold higher than that in the control group $(3.04 \pm 3.26 \mathrm{ng} / \mathrm{mL})$ (Figure 3C).

Patients without CKD and with CKD were also analyzed separately. In patients without CKD in stage IIa, CI-AKI occurred in $17(3.2 \%)$ patients. Plasma exposure of RST and DM-RST, dosage, hypertension, level of $\mathrm{CK}$, and $\mathrm{CKMB}$ were entered into the multivariate logistic model, and only plasma RST exposure (OR: 3.556; 95\% CI: 1.763-7.171; $p=0.0004$ ), dosage (OR: 1.083 ; 95\% CI: $1.000-1.173 ; p=0.0493$ ), and hypertension (OR: 3.492; 95\% CI: $1.167-10.450 ; p=0.0253$ ) were retained in the model implying that they were independent risk factors for CI-AKI (Table S3). CI-AKI patients $(7.61 \pm 4.66 \mathrm{ng} / \mathrm{mL})$ exhibited 2.5 fold higher plasma RST concentration than controls (3.04 \pm $3.26 \mathrm{ng} / \mathrm{mL}$ ) (Figure 3A).

In patients with $\mathrm{CKD}$ in stage IIb, CI-AKI occurred in 13 (12.5\%) patients. Higher plasma exposure of RST and DMRST were associated with a higher risk of CI-AKI. Multivariate analysis revealed that patients in the CI-AKI group had higher plasma DM-RST exposure (OR: 1.935; 95\% CI: 1.056-3.547; $p=0.0327$ ), and were with more heart failure (OR: 18.817; $95 \%$ 
TABLE 1 | Patient characteristics and their effects on plasma concentration of AT-all.

\begin{tabular}{|c|c|c|c|c|c|c|c|}
\hline \multirow[t]{3}{*}{ Characteristics } & & \multirow[t]{3}{*}{ Value $N(\%)$ or mean $\pm S D$} & \multicolumn{5}{|c|}{ Plasma AT-all concentration, $\mathrm{ng} / \mathrm{mL}$} \\
\hline & & & \multirow[b]{2}{*}{ mean $\pm S D$} & \multicolumn{2}{|c|}{ Univariate analysis } & \multicolumn{2}{|c|}{ Multivariate analysis } \\
\hline & & & & Estimate & $p$-value & Estimate & $p$-value \\
\hline \multicolumn{8}{|l|}{ DEMOGRAPHIC DATA } \\
\hline Total number & & 1219 & $9.25 \pm 11.19$ & & & & \\
\hline Age (years) & & $62.95 \pm 10.00$ & & 0.0122 & $<0.0001$ & 0.0140 & $<0.0001$ \\
\hline \multirow[t]{2}{*}{ Sex } & Female & $297(24.36)$ & $9.89 \pm 14.40$ & -0.0032 & 0.9633 & & \\
\hline & Male & $922(75.64)$ & $9.04 \pm 9.94$ & & & & \\
\hline \multirow[t]{3}{*}{ Dosage (mg) } & 10 & $21(1.72)$ & $4.23 \pm 3.69$ & 0.0197 & $<0.0001$ & 0.0173 & $<0.0001$ \\
\hline & 20 & 1058 (86.79) & $8.72 \pm 10.47$ & & & & \\
\hline & 40 & $140(11.48)$ & $13.98 \pm 15.27$ & & & & \\
\hline SYNTAX score & & $13.81 \pm 12.19$ & & 0.0094 & 0.0001 & 0.0059 & 0.0161 \\
\hline \multicolumn{8}{|l|}{ MEDICAL HISTORY } \\
\hline \multirow[t]{2}{*}{ Arrhythmia } & No & $1104(90.86)$ & $9.08 \pm 10.61$ & 0.0707 & 0.4914 & & \\
\hline & Yes & $111(9.14)$ & $10.98 \pm 15.86$ & & & & \\
\hline \multirow[t]{2}{*}{ Diabetes } & No & 895 (73.66) & $8.99 \pm 10.56$ & 0.0266 & 0.6924 & & \\
\hline & Yes & $320(26.34)$ & $9.99 \pm 12.82$ & & & & \\
\hline \multirow[t]{2}{*}{ Heart failure } & No & 1097 (90.29) & $8.93 \pm 10.46$ & 0.1210 & 0.2261 & & \\
\hline & Yes & $118(9.71)$ & $12.24 \pm 16.35$ & & & & \\
\hline \multirow[t]{2}{*}{ Hypertension } & No & $499(41.04)$ & $8.40 \pm 9.14$ & 0.0881 & 0.1435 & & \\
\hline & Yes & $717(58.96)$ & $9.83 \pm 12.41$ & & & & \\
\hline \multirow[t]{2}{*}{ Hyperlipidemia } & No & $1073(88.24)$ & $9.36 \pm 11.28$ & -0.0265 & 0.7734 & & \\
\hline & Yes & $143(11.76)$ & $8.40 \pm 10.57$ & & & & \\
\hline \multicolumn{8}{|c|}{ BIOCHEMICAL MEASUREMENTS } \\
\hline ALT, U/L & & $28.69 \pm 16.80$ & & 0.0055 & 0.0017 & 0.0059 & 0.0012 \\
\hline AST, U/L & & $31.05 \pm 30.42$ & & 0.0031 & 0.0014 & & \\
\hline Scr, $\mu \mathrm{mol} / \mathrm{L}$ & & $96.70 \pm 81.69$ & & 0.0014 & $<0.0001$ & 0.0012 & 0.0008 \\
\hline eGFR, $\mathrm{mL} / \mathrm{min} / 1.73 \mathrm{~m}^{2}$ & & $91.26 \pm 72.98$ & & -0.0013 & 0.0013 & & \\
\hline CK, U/L & & $158.91 \pm 408.68$ & & 0.0001 & 0.4328 & & \\
\hline CKMB, U/L & & $8.82 \pm 13.67$ & & 0.0026 & 0.2603 & & \\
\hline $\mathrm{CHOL}, \mathrm{mmol} / \mathrm{L}$ & & $4.32 \pm 1.13$ & & 0.0318 & 0.2305 & & \\
\hline LDLC, mmol/L & & $2.60 \pm 0.94$ & & 0.0569 & 0.0739 & & \\
\hline HDLC, mmol/L & & $0.99 \pm 0.26$ & & -0.1445 & 0.2058 & & \\
\hline TRIG, mmol/L & & $1.58 \pm 1.11$ & & -0.0018 & 0.9463 & & \\
\hline GLUC, mmol/L & & $6.66 \pm 2.59$ & & 0.0267 & 0.0204 & & \\
\hline Lpa, mg/L & & $295.05 \pm 315.79$ & & 0.0003 & 0.0114 & & \\
\hline APOA, g/L & & $1.06 \pm 0.29$ & & -0.3237 & 0.0048 & & \\
\hline \multicolumn{8}{|l|}{ MEDICATION } \\
\hline \multirow[t]{2}{*}{$\beta$-blockers } & No & $137(11.27)$ & $8.20 \pm 8.00$ & 0.0263 & 0.7787 & & \\
\hline & Yes & 1079 (88.73) & $9.38 \pm 11.54$ & & & & \\
\hline \multirow[t]{2}{*}{ ACEls } & No & $467(38.4)$ & $9.46 \pm 11.17$ & -0.0255 & 0.6761 & & \\
\hline & Yes & $749(61.6)$ & $9.11 \pm 11.22$ & & & & \\
\hline \multirow[t]{2}{*}{ CCBs } & No & $848(69.74)$ & $8.93 \pm 10.17$ & -0.0102 & 0.8743 & & \\
\hline & Yes & $368(30.26)$ & $9.97 \pm 13.25$ & & & & \\
\hline \multirow[t]{2}{*}{ PPls } & No & $588(48.36)$ & $8.69 \pm 10.79$ & 0.0550 & 0.3535 & & \\
\hline & Yes & 628 (51.64) & $9.77 \pm 11.55$ & & & & \\
\hline
\end{tabular}

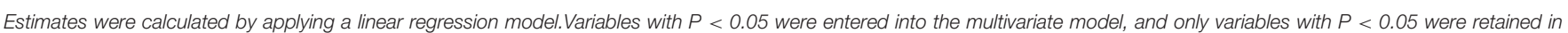

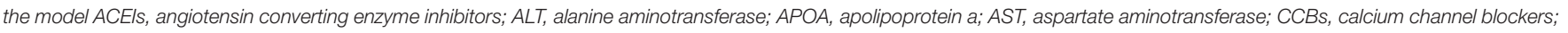

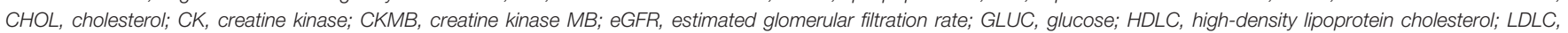

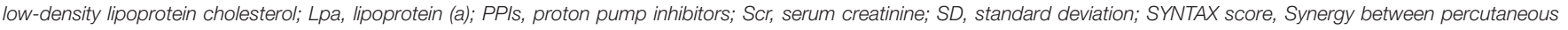
coronary intervention with TAXUS and Cardiac Surgery score; TRIG, triglyceride. 
TABLE 2 | Patient characteristics and their effects on plasma concentration of RST.

\begin{tabular}{|c|c|c|c|c|c|c|c|}
\hline \multirow[t]{2}{*}{ Characteristics } & & \multirow[t]{2}{*}{ Value $\boldsymbol{N}(\%)$ or mean $\pm S D$} & \multicolumn{5}{|c|}{ Plasma RST concentration, $\mathrm{ng} / \mathrm{mL}$} \\
\hline & & & mean $\pm S D$ & Estimate & $p$-value & Estimate & $p$-value \\
\hline \multicolumn{8}{|l|}{ DEMOGRAPHIC DATA } \\
\hline Total number & & 635 & $3.29 \pm 3.57$ & & & & \\
\hline & Male & $471(74.17)$ & $3.16 \pm 3.51$ & & & & \\
\hline \multirow[t]{4}{*}{ Dosage (mg) } & 5 & $11(1.74)$ & $2.79 \pm 3.64$ & -0.0052 & 0.7932 & & \\
\hline & 10 & 549 (86.73) & $3.19 \pm 3.42$ & & & & \\
\hline & 20 & $67(10.58)$ & $4.32 \pm 4.57$ & & & & \\
\hline & 40 & $6(0.95)$ & $2.40 \pm 3.53$ & & & & \\
\hline Arrhythmia & Yes & $51(8.03)$ & $4.32 \pm 4.73$ & & & & \\
\hline \multirow[t]{2}{*}{ Diabetes } & No & $503(79.21)$ & $3.11 \pm 3.33$ & 0.2358 & 0.2498 & & \\
\hline & Yes & $132(20.79)$ & $3.97 \pm 4.32$ & & & & \\
\hline \multirow[t]{2}{*}{ Heart failure } & No & $591(93.07)$ & $3.14 \pm 3.29$ & 0.0189 & 0.9539 & & \\
\hline & Yes & $44(6.93)$ & $5.34 \pm 5.90$ & & & & \\
\hline \multirow[t]{2}{*}{ Hypertension } & No & $356(56.06)$ & $3.21 \pm 3.50$ & 0.0603 & 0.7189 & & \\
\hline & Yes & 279 (43.94) & $3.40 \pm 3.67$ & & & & \\
\hline \multirow[t]{2}{*}{ Hyperlipidemia } & No & $566(89.13)$ & $3.27 \pm 3.57$ & 0.1139 & 0.6699 & & \\
\hline & Yes & $69(10.87)$ & $3.45 \pm 3.63$ & & & & \\
\hline \multicolumn{8}{|c|}{ BIOCHEMICAL MEASUREMENTS } \\
\hline LDLC, mmol/L & & $2.77 \pm 1.13$ & & -0.2219 & 0.0029 & & \\
\hline HDLC, mmol/L & & $1.00 \pm 0.26$ & & -0.3090 & 0.3473 & & \\
\hline TRIG, mmol/L & & $1.67 \pm 1.24$ & & 0.0994 & 0.1407 & & \\
\hline GLUC, mmol/L & & $7.04 \pm 3.20$ & & -0.0251 & 0.3342 & & \\
\hline Lpa, mg/L & & $275.42 \pm 292.45$ & & -0.0006 & 0.0658 & & \\
\hline APOA, g/L & & $1.06 \pm 0.27$ & & 0.1348 & 0.6887 & & \\
\hline \multicolumn{8}{|l|}{ MEDICATION } \\
\hline \multirow[t]{2}{*}{$\beta$-blockers } & No & 86 (13.54) & $3.71 \pm 4.11$ & -0.3353 & 0.1675 & & \\
\hline & Yes & 549 (86.46) & $3.22 \pm 3.48$ & & & & \\
\hline \multirow[t]{2}{*}{ ACEls } & No & 284 (44.72) & $3.54 \pm 3.59$ & -0.3823 & 0.0220 & -0.3584 & 0.0320 \\
\hline & Yes & 351 (55.28) & $3.09 \pm 3.55$ & & & & \\
\hline \multirow[t]{2}{*}{ CCBs } & No & 455 (71.65) & $3.35 \pm 3.70$ & -0.0735 & 0.6904 & & \\
\hline & Yes & 180 (28.35) & $3.15 \pm 3.24$ & & & & \\
\hline \multirow[t]{2}{*}{ PPls } & No & 288 (45.35) & $3.04 \pm 3.18$ & 0.1108 & 0.5070 & & \\
\hline & Yes & 347 (54.65) & $3.49 \pm 3.86$ & & & & \\
\hline
\end{tabular}

Estimates were calculated by applying a linear regression model. Variables with $P<0.05$ were entered into the multivariate model, and only variables with $P<0.05$ were retained in the model.

RST, rosuvastatin; other abbreviations as in Table 1. 
TABLE 3 | Effects of baseline characteristics and plasma concentrations of AT and its metabolites on Cl-AKI in stage I.

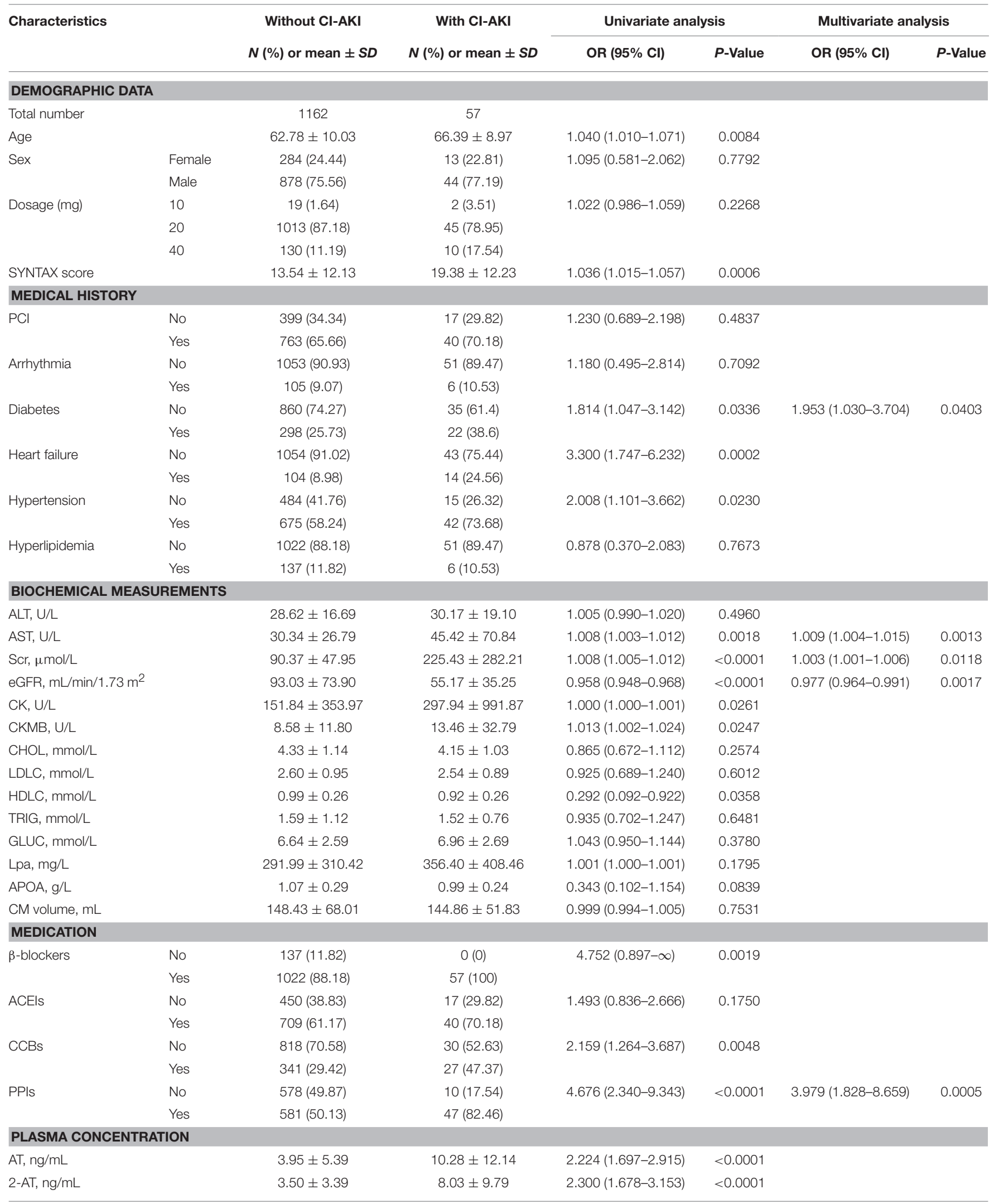


TABLE 3 | Continued

\begin{tabular}{|c|c|c|c|c|c|c|}
\hline \multirow[t]{2}{*}{ Characteristics } & \multirow{2}{*}{$\begin{array}{c}\text { Without CI-AKI } \\
N(\%) \text { or mean } \pm S D\end{array}$} & \multirow{2}{*}{$\begin{array}{c}\text { With CI-AKI } \\
N(\%) \text { or mean } \pm S D\end{array}$} & \multicolumn{2}{|c|}{ Univariate analysis } & \multicolumn{2}{|c|}{ Multivariate analysis } \\
\hline & & & OR (95\% Cl) & $P$-Value & OR (95\% Cl) & $P$-Value \\
\hline 4-AT, ng/mL & $1.28 \pm 1.94$ & $4.43 \pm 5.95$ & $2.503(1.942-3.226)$ & $<0.0001$ & & \\
\hline ATL, ng/mL & $3.91 \pm 6.02$ & $10.11 \pm 12.06$ & 1.959 (1.533-2.503) & $<0.0001$ & & \\
\hline 2-ATL, ng/mL & $8.94 \pm 9.70$ & $17.58 \pm 14.77$ & $2.033(1.518-2.724)$ & $<0.0001$ & & \\
\hline 4-ATL, ng/mL & $1.66 \pm 2.32$ & $4.03 \pm 5.24$ & $2.246(1.721-2.932)$ & $<0.0001$ & & \\
\hline AT-all, ng/mL & $8.60 \pm 9.65$ & $22.40 \pm 24.63$ & $2.826(2.075-3.851)$ & $<0.0001$ & 2.265 (1.609-3.187) & $<0.0001$ \\
\hline
\end{tabular}

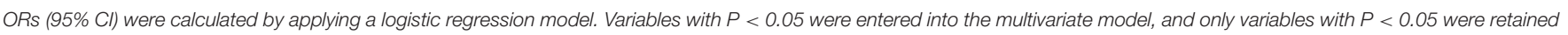
in the model.

2-AT, 2-hydroxy atorvastatin; 2-ATL, 2-hydroxy atorvastatin lactone; 4-AT, 4-hydroxy atorvastatin; 4-ATL, 4-hydroxy atorvastatin lactone; AT, atorvastatin; ATL, atorvastatin lactone; Cl,

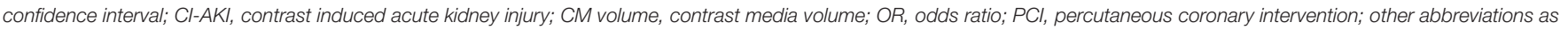
in Table 1.

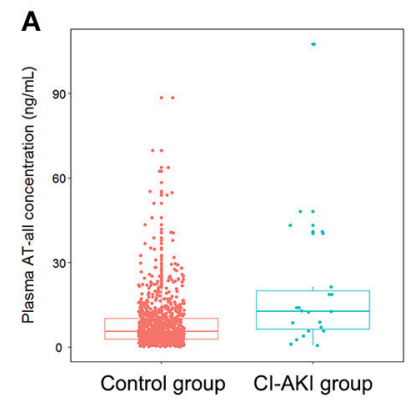

D

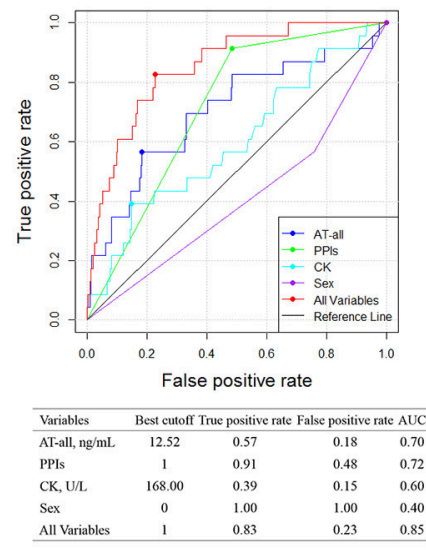

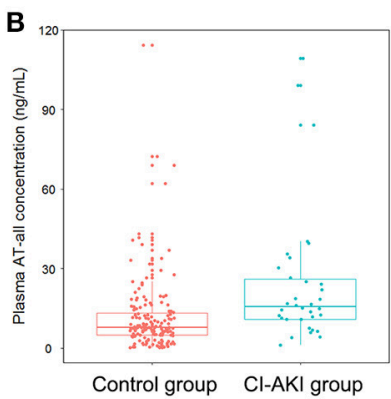

$\mathbf{E}$

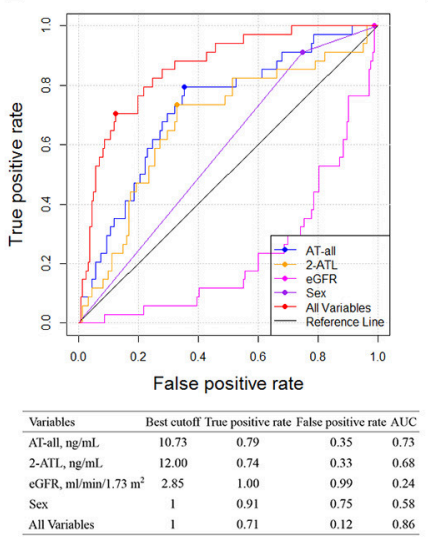

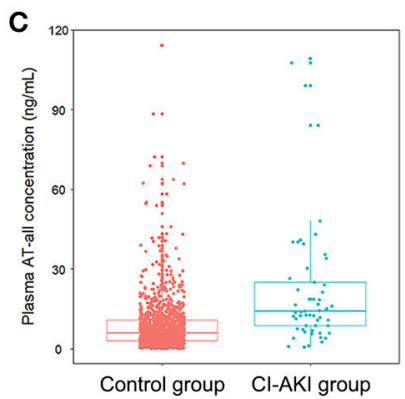

$\mathbf{F}$

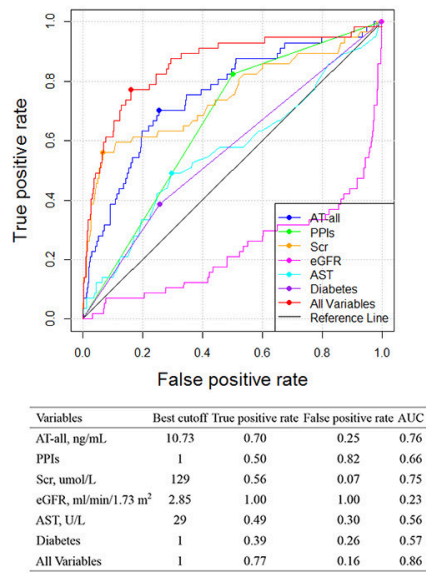

FIGURE 2 | Comparison of plasma AT-all concentration between control group and Cl-AKI group in patients without CKD (A), in patients with CKD (B), and in all patients (C) in stage I. ROC analyses of variables for predicting CI-AKI in patients without CKD (D), in patients with CKD (E), and in all patients (F) in stage I. 2-ATL, 2-hydroxy atorvastatin lactone; AST, aspartate aminotransferase; CK, creatine kinase; eGFR, estimated glomerular filtration rate; PPIs, proton pump inhibitors; ROC, receiver operating characteristic; Scr, serum creatinine; other abbreviations as in Figure 1.

CI: 4.334-81.701; $p<0.0001)$ (Table S4). CI-AKI patients $(9.15 \pm$ $6.51 \mathrm{ng} / \mathrm{mL}$ ) exhibited 3.0-fold higher plasma RST concentration than controls $(3.05 \pm 3.28 \mathrm{ng} / \mathrm{mL})$ (Figure 3B).

\section{Predictive Diagnostic Power of Plasma AT and RST Exposure for CI-AKI}

To better predict occurrence of CI-AKI, we developed a prognostic classifier combining clinical characteristics and plasma concentrations. In stage I, the AUCs of AT-all, in groups without and with CKD, performed similar characteristics (0.70 and 0.73 , respectively) (Figures 2D,E). The AUC of plasma ATall exposure for all patients in stage I was 0.76. A cutoff of $10.73 \mathrm{ng} / \mathrm{mL}$ performed high true positive rate (70\%) and low false positive rate $(25 \%)$ (Figure 2 F). Predictive value of use of PPIs, diabetes, level of Scr, AST, and eGFR for CI-AKI, as determined by the ROC curves, were insufficient with AUC varying between 0.23 and 0.75 . However, predictive effectiveness of combined variables was substantially increased to 0.86 . 
TABLE 4 | Effects of baseline characteristics and plasma concentrations of RST and its metabolites on CI-AKI in stage II.

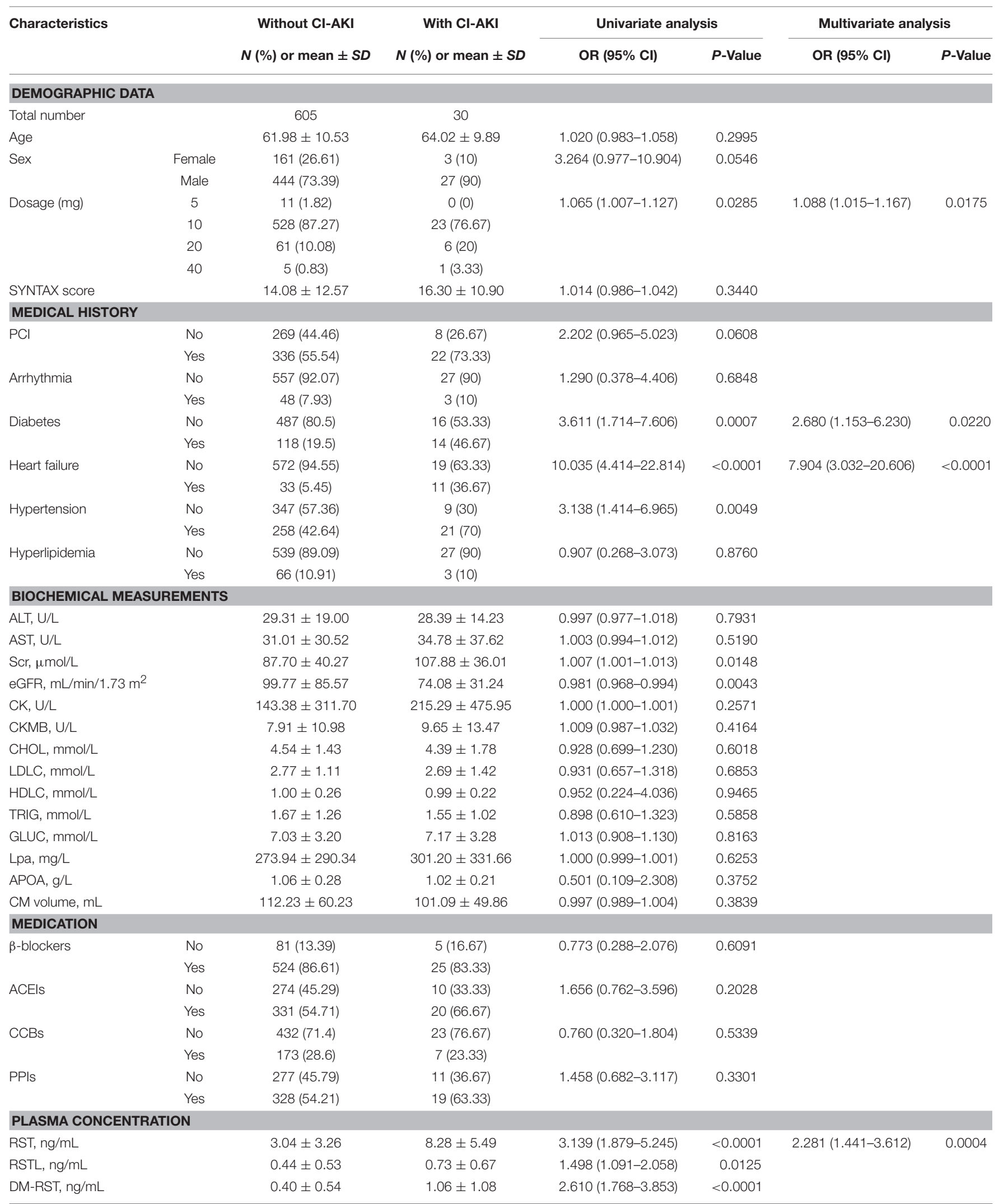

Variables with $P<0.05$ were entered into the multivariate model, and only variables with $P<0.05$ were retained in the model. Abbreviations as in Tables 1-3. 

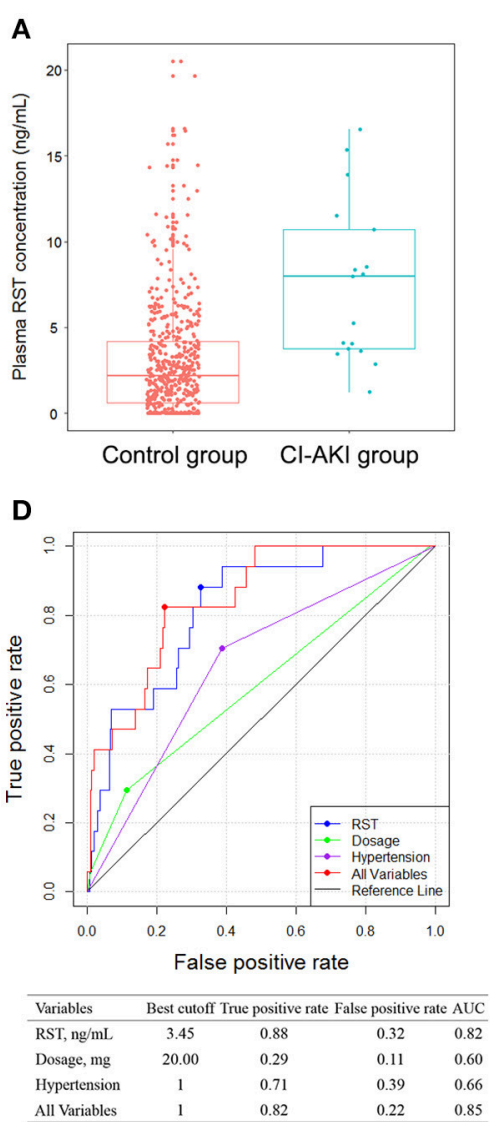
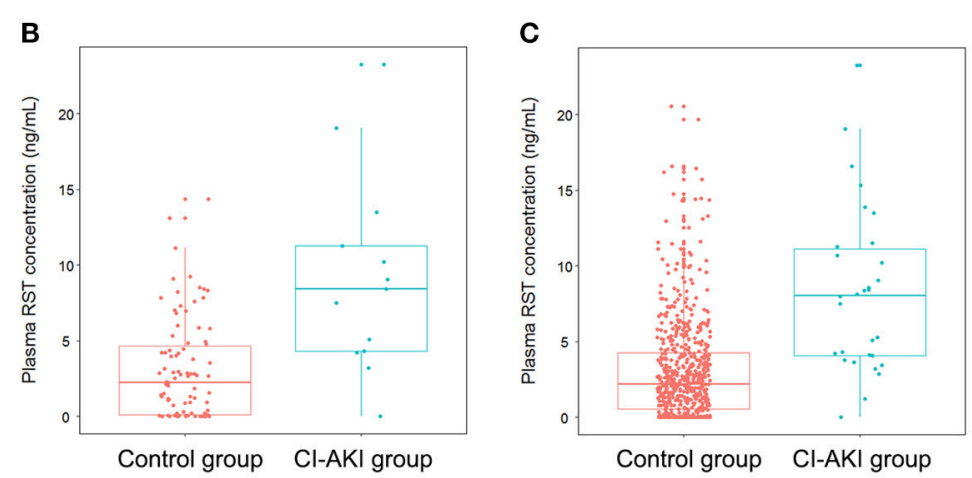

E

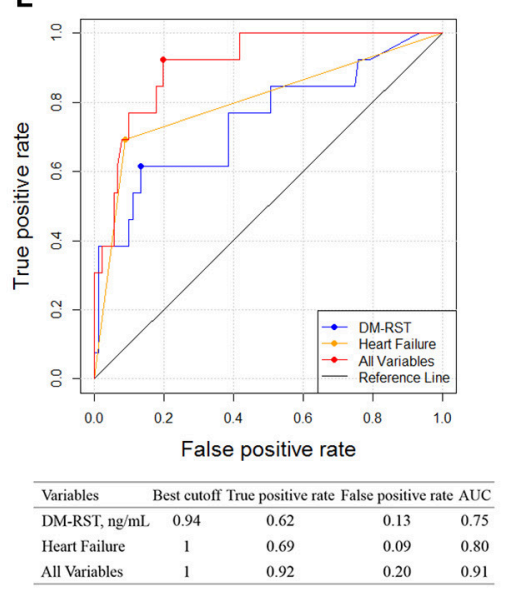

F

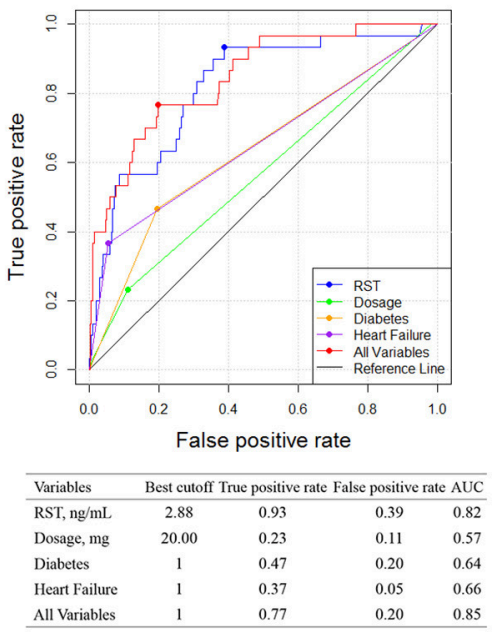

FIGURE 3 | Comparison of plasma RST concentration between control group and Cl-AKI group in patients without CKD (A), in patients with CKD (B), and in all patients (C) in stage II. ROC analyses of variables for predicting Cl-AKI in patients without CKD (D), in patients with CKD (E), and in all patients (F) in stage II. DM-RST: N-desmethyl rosuvastatin, RST: rosuvastatin; other abbreviations as in Figures 1, 2.

AUC for predicting CI-AKI was calculated in an additional independent cohort that receiving RST treatment (stage II study, Figures 3D,E). As shown in Figure 3F, plasma RST exposure was validated in predicting CI-AKI in the stage II cohort (AUC $=0.82$ ). Moreover, the best cutoff of plasma RST exposure was $2.88 \mathrm{ng} / \mathrm{mL}$, and the predictive performance of plasma RST exposure in the validation set yielded high true positive rate (93\%) and low false positive rate (39\%). After integrating all variable, AUC for the CI-AKI classifier was 0.85 .

\section{DISCUSSION}

Statins are one of the most commonly prescribed medications all over the world, rendering it important to determine their potentially effects in CI-AKI. To the best of our knowledge, this is the first study to investigate the risk of CI-AKI in relation to high plasma statin exposure in a prospective study of patients with CAD. Our study provided solid evidence that high plasma statins exposure independently increased the risk of CIAKI, after adjusting for several potential confounding variables, including demographics, severity of CAD, clinical measurements, prevalent comorbidities, and concomitant use of medications.
These findings have important implications for the management of statins therapy in patients undergoing CAG.

Additionally, this study identified that the use of PPIs was significantly and independently associated with increased risk of CI-AKI, and should be administered carefully. These findings are also in agreement with studies reporting (Arora et al., 2016; Lazarus et al., 2016; Moledina and Perazella, 2016) that PPIs are emerging as an important contributing cause to CKD. Clinicians should closely monitor patients taking PPIs by urinalysis and renal function tests to recognize any renal insufficiency in time.

With the increasing role of contrast media in diagnostic and interventional procedures, especially in the field of cardiology, the prevalence of CI-AKI is expected to rise. Pathophysiology of CI-AKI is not exactly understood, and multiple causes, including acute tubular necrosis from poor perfusion, nephrotoxicity from contrast media, use of nephrotoxic medications, cholesterol embolization, procedure-related factors, or a combination of these, may be involved (Kooiman et al., 2015). The prevention of CI-AKI can decrease mortality, morbidity, therapeutic costs and hospital stays. The role of various drugs in prevention of CIAKI (such as statins) is still controversial and warrants future studies. Some researchers have reported no association between 
statin use and CI-AKI, whereas others have found that statin use is protective against the incidence of CI-AKI, or that it is associated with an increased risk of CI-AKI. In 2012, Li et al. (2012) performed a meta-analysis including 7 studies and 1,399 patients that investigated the potential benefit of short-term highdose statins in the prevention of CI-AKI. The results of this study demonstrated that a significant improvement in the incidence of CI-AKI. On the contrary, it has been reported that a shortterm administration of high doses of atorvastatin before and after contrast exposure does not decrease the incidence of contrastinduced nephropathy in patients with pre-existing CKD (Toso et al., 2010). One potential factor contributing to this discrepancy is the presence of marked clinical and statistical heterogeneity between studies.

Our results confirm the findings from previous retrospective observational analysis (Dormuth et al., 2013) and retrospective cohort study (Chung et al., 2013). The former discovered that in patients with non-chronic kidney disease, current users of highpotency statins were $34 \%$ more likely to be hospitalized with AKI within 120 days after starting treatment (Dormuth et al., 2013). The latter found that statins with high cholesterol-lowering efficacy might increase the risk for developing severe renal failure (Chung et al., 2013). The biological mechanism of statins on kidney injury remained not fully investigated. The higher risk of CI-AKI in patients with high potency statins treatment may be related to an increased risk of proteinuria or rhabdomyolysis. Our results was also supported by an experimental study that AT with a dose of $150 \mathrm{mg} / \mathrm{kg} /$ day for 7 days was nephrotoxic for rats, whereas lower doses at 10 or $50 \mathrm{mg} / \mathrm{kg} /$ day for 7 days were not accompanied by renal injury (Nasri et al., 2016), suggesting that administration of statins in high doses may itself be directed to renal tubular cell injury.

CI-AKI is likely to remain a significant challenge for cardiologists in the future because the prevalence of comorbid conditions with aging among patients with CKD. It is more frequent in elderly patients with renal insufficiency. Some authors agreed that when renal function is normal, there is no risk of CI-AKI (Andreucci et al., 2017). Therefore, we further divided patients into two subgroups according to baseline renal function, and discovered that patients with CKD had higher incidence than that in patients without CKD. The association between high plasma statin exposure and CI-AKI still remained highly significant in both subgroups.

Several limitations of this study need to be mentioned. Firstly, it was a single-center study and the sample size

\section{REFERENCES}

Andreucci, M., Faga, T., Serra, R., De Sarro, G., and Michael, A. (2017). Update on the renal toxicity of iodinated contrast drugs used in clinical medicine. Drug Healthc. Patient Saf. 9, 25-37. doi: 10.2147/DHPS.S1 22207

Argalious, M., Xu, M., Sun, Z., Smedira, N., and Koch, C. G. (2010). Preoperative statin therapy is not associated with a reduced incidence of postoperative acute kidney injury after cardiac surgery. Anesth. Analg. 111, 324-330. doi: 10.1213/ANE.0b013e3181d8a078 was relatively small. To address this defect, we used other patient populations that receiving other statin treatment to validate and confirm the predictive value of plasma statin and its metabolites concentrations for CI-AKI. Secondly, we detected the statin concentration at $10-12 \mathrm{~h}$ after dose instead of whole profile. However, in the clinical setting, it is easier to monitor steady statin concentration. Additional large-scale, multicenter clinical trial of statin efficacy in CAD patients is thus warranted.

\section{CONCLUSION}

Our study demonstrated that high plasma exposure of AT and its metabolites could significantly increase the risk of CI-AKI, which was further validated and confirmed in patients receiving RST treatment. Thus, statins should be used with greater caution in patients with CAD undergoing CAG, and plasma levels of statin and metabolites should be monitored to reduce the occurrence of CI-AKI.

\section{AUTHOR CONTRIBUTIONS}

LC, XB, HL and HW performed experiment, performed data analysis, and wrote the manuscript; QZ, ShaZ and YiL participated in data analysis; YoL, GH participated in patient recruitment; QL, JC and BZ revised manuscript; ShiZ, MH and QG designed the study and revised manuscript. All authors reviewed and approved the final manuscript.

\section{ACKNOWLEDGMENTS}

This work was supported by the National key R\&D program (No. 2017YFC0909301, 2016YFC0905003), National Nature Science Foundation of China (No. 81673514, 81373486, 81202602), Science and Technology Development Projects of Guangdong Province, China (No. 2016B090918114), and Science and Technology Development Projects of Guangzhou, Guangdong, China (201510010236, 201604020096).

\section{SUPPLEMENTARY MATERIAL}

The Supplementary Material for this article can be found online at: https://www.frontiersin.org/articles/10.3389/fphar. 2018.00427/full\#supplementary-material

Arora, P., Gupta, A., Golzy, M., Patel, N., Carter, R. L., Jalal, K., et al. (2016). Proton pump inhibitors are associated with increased risk of development of chronic kidney disease. BMC Nephrol. 17:112. doi: 10.1186/s12882-016-0325-4

Aurelio, A., and Durante, A. (2014). Contrast-induced nephropathy in percutaneous coronary interventions: pathogenesis, risk factors, outcome, prevention and treatment. Cardiology 128, 62-72. doi: 10.1159/000358042

Billings, F. T., Hendricks, P. A., Schildcrout, J. S., Shi, Y., Petracek, M. R., Byrne, J. G., et al. (2016). High-dose perioperative atorvastatin and acute kidney injury following cardiac surgery: a randomized clinical trial. JAMA 315, 877-888. doi: 10.1001/jama.2016.0548 
Birnie, K., Verheyden, V., Pagano, D., Bhabra, M., Tilling, K., Sterne, J. A., et al. (2014). UK AKI in Cardiac Surgery Collaborators. Predictive models for kidney disease: improving global outcomes (KDIGO) defined acute kidney injury in UK cardiac surgery. Crit. Care 18:606. doi: 10.1186/s13054-014-0606-X

Cai, L. Y., Zheng, Z. J., Wang, X. P., Tang, L., Mai, L. P., He, G. D., et al. (2017). Simultaneous determination of atorvastatin and its metabolites in human plasma by UPLC-MS/MS. Anal. Methods 9, 1038-1045. doi: 10.1039/C6AY03113G

Chalikias, G., Drosos, I., and Tziakas, D. N. (2016). Prevention of contrastinduced acute kidney injury: an update. Cardiovasc. Drugs Ther. 30, 515-524. doi: 10.1007/s10557-016-6683-0

Chung, Y. H., Lee, Y. C., Chang, C. H., Lin, M. S., Lin, J. W., and Lai, M. S. (2013). Statins of high versus low cholesterol-lowering efficacy and the development of severe renal failure. Pharmacoepidemiol. Drug Saf. 22, 583-592. doi: $10.1002 /$ pds.3433

DeGorter, M. K., Tirona, R. G., Schwarz, U. I., Choi, Y. H., Dresser, G. K., Suskin, N., et al. (2013). Clinical and pharmacogenetic predictors of circulating atorvastatin and rosuvastatin concentrations in routine clinical care. Circ. Cardiovasc. Genet. 6, 400-408. doi: 10.1161/CIRCGENETICS.113.000099

Dormuth, C. R., Hemmelgarn, B. R., Paterson, J. M., James, M. T., Teare, G. F., Raymond, C. B., et al. (2013). Canadian network for observational drug effect studies. Use of high potency statins and rates of admission for acute kidney injury: multicenter, retrospective observational analysis of administrative databases. BMJ 346:f880. doi: 10.1136/bmj.f880

Fihn, S. D., Blankenship, J. C., Alexander, K. P., Bittl, J. A., Byrne, J. G., Fletcher, B. J., et al. (2014). ACC/AHA/AATS/PCNA/SCAI/STS focused update of the guideline for the diagnosis and management of patients with stable ischemic heart disease: a report of the American College of Cardiology/American Heart Association Task Force on practice guidelines, and the American Association for Thoracic Surgery, Preventive Cardiovascular Nurses Association, Society for Cardiovascular Angiography and Interventions, and Society of Thoracic Surgeons. J. Am. Coll. Cardiol. 64, 1929-1949. doi: 10.1016/j.jacc.2014.07.017

Goldenberg, I., and Matetzky, S. (2005). Nephropathy induced by contrast media: pathogenesis, risk factors and preventive strategies. CMAJ 172, 1461-1471. doi: $10.1503 / \mathrm{cmaj} .1040847$

Ikeda, N., Kogame, N., Iijima, R., Nakamura, M., and Sugi, K. (2012). Carotid artery intima-media thickness and plaque score can predict the SYNTAX score Eur. Heart J. 33, 113-119. doi: 10.1093/eurheartj/ehr399

Itoh, Y., Yano, T., Sendo, T., and Oishi, R. (2005). Clinical and experimental evidence for prevention of acute renal failure induced by radiographic contrast media. J. Pharmacol. Sci. 97, 473-488. doi: 10.1254/jphs.CRJ05002X

Khanal, S., Attallah, N., Smith, D. E., Kline-Rogers, E., Share, D., O’Donnell, M. J., et al. (2005). Statin therapy reduces contrast-induced nephropathy: an analysis of contemporary percutaneous interventions. Am. J. Med. 118, 843-849. doi: 10.1016/j.amjmed.2005.03.031

National Kidney Foundation (2002). K/DOQI clinical practice guidelines for chronic kidney disease: evaluation, classification, and stratification. Am. J. Kidney Dis. 39, S1-S266.

Kooiman, J., Seth, M., Nallamothu, B. K., Heung, M., Humes, D., and Gurm, H. S. (2015). Association between acute kidney injury and in-hospital mortality in patients undergoing percutaneous coronary interventions. Circ. Cardiovasc. Interv. 81:e002212. doi: 10.1161/CIRCINTERVENTIONS.114.002212

Lazarus, B., Chen, Y., Wilson, F. P., Sang, Y., Chang, A. R., Coresh, J., et al. (2016). Proton pump inhibitor use and the risk of chronic kidney disease. JAMA Intern. Med. 176, 238-246. doi: 10.1001/jamainternmed.2015.7193

Ledneva, E., Karie, S., Launay-Vacher, V., Janus, N., and Deray, G. (2009). Renal safety of gadolinium-based contrast media in patients with chronic renal insufficiency. Radiology 250, 618-628. doi: 10.1148/radiol.2503080253

Lennernas, H. (2003). Clinical pharmacokinetics of atorvastatin. Clin. Pharmacokinet. 42, 1141-1160. doi: 10.2165/00003088-200342130-00005

Lev, E. I., Kornowski, R., Vaknin-Assa, H., Ben-Dor, I., Brosh, D., Teplitsky, I., et al. (2009). Effect of previous treatment with statins on outcome of patients with ST-segment elevation myocardial infarction treated with primary percutaneous coronary intervention. Am. J. Cardiol. 103, 165-169. doi: 10.1016/j.amjcard.2008.08.052

Li, Y., Liu, Y., Fu, L., Mei, C., and Dai, B. (2012). Efficacy of shortterm high-dose statin in preventing contrast-induced nephropathy: a meta-analysis of seven randomized controlled trials. PLoS ONE 7:e34450. doi: 10.1371/journal.pone.0034450
Mehta, R. L., Kellum, J. A., Shah, S. V., Molitoris, B. A., Ronco, C., Warnock, D. G., et al. (2007). Acute Kidney Injury Network: report of an initiative to improve outcomes in acute kidney injury. Crit. Care 11:R31. doi: 10.1186/cc5713

Mithani, S., Kuskowski, M., Slinin, Y., Ishani, A., McFalls, E., and Adabag, S. (2011). Dose-dependent effect of statins on the incidence of acute kidney injury after cardiac surgery. Ann. Thorac. Surg. 91, 520-525. doi: 10.1016/j.athoracsur.2010.10.061

Mohr, F. W., Morice, M. C., Kappetein, A. P., Feldman, T. E., Ståhle, E., Colombo, A., et al. (2013). Coronary artery bypass graft surgery versus percutaneous coronary intervention in patients with three-vessel disease and left main coronary disease: 5 -year follow-up of the randomised, clinical SYNTAX trial. Lancet 381, 629-638. doi: 10.1016/S0140-6736(13)60141-5

Moledina, D. G., and Perazella, M. A. (2016). PPIs and kidney disease: from AIN to CKD. J. Nephrol. 29, 611-616. doi: 10.1007/s40620-016-0309-2

Nash, K., Hafeez, A., and Hou, S. (2002). Hospital-acquired renal insufficiency. Am. J. Kidney Dis. 39, 930-936. doi: 10.1053/ajkd.2002.32766

Nasri, H., Hasanpour, Z., Nematbakhsh, M., Ahmadi, A., and Rafieian-Kopaei, M. (2016). The effect of the various doses of atorvastatin on renal tubular cells an experimental study. J. Nephropathol. 5, 111-115. doi: 10.15171/jnp.2016.20

Park, J. H., Shim, J. K., Song, J. W., Soh, S., and Kwak, Y. L. (2016). Effect of atorvastatin on the incidence of acute kidney injury following valvular heart surgery: a randomized, placebo-controlled trial. Intensive Care Med. 42, 1398-1407. doi: 10.1007/s00134-016-4358-8

Patti, G., Nusca, A., Chello, M., Pasceri, V., D’Ambrosio, A., Vetrovec, G. W., et al. (2008). Usefulness of statin pretreatment to prevent contrastinduced nephropathy and to improve long-term outcome in patients undergoing percutaneous coronary intervention. Am. J. Cardiol. 101, 279-285. doi: 10.1016/j.amjcard.2007.08.030

Patti, G., Ricottini, E., Nusca, A., Colonna, G., Pasceri, V., D’Ambrosio, A., et al. (2011). Short-term, high-dose atorvastatin pretreatment to prevent contrastinduced nephropathy in patients with acute coronary syndromes undergoing percutaneous coronary intervention (from the ARMYDA-CIN [atorvastatin for reduction of myocardial damage during angioplasty-contrast-induced nephropathy] trial. Am. J. Cardiol. 108, 1-7. doi: 10.1016/j.amjcard.2011. 03.001

Prasad, A., Ortiz-Lopez, C., Khan, A., Levin, D., and Kaye, D. M. (2016). Acute kidney injury following peripheral angiography and endovascular therapy: a systematic review of the literature. Catheter. Cardiovasc. Interv. 88, 264-273. doi: $10.1002 / \mathrm{ccd} .26466$

Serruys, P. W., Morice, M. C., Kappetein, A. P., Colombo, A., Holmes, D. R., Mack, M. J., et al. (2009). Percutaneous coronary intervention versus coronary-artery bypass grafting for severe coronary artery disease. N. Engl. J. Med. 360, 961-972. doi: 10.1056/NEJMoa0804626

Sianos, G., Morel, M. A., Kappetein, A. P., Morice, M. C., Colombo, A., Dawkins, K., et al. (2005). The SYNTAX score: an angiographic tool grading the complexity of coronary artery disease. EuroIntervention 1, 219-227.

Toso, A., Maioli, M., Leoncini, M., Gallopin, M., Tedeschi, D., Micheletti, C., et al. (2010). Usefulness of atorvastatin $(80 \mathrm{mg})$ in prevention of contrastinduced nephropathy in patients with chronic renal disease. Am. J. Cardiol. 105, 288-292. doi: 10.1016/j.amjcard.2009.09.026

White, C. M. (2002). A review of the pharmacologic and pharmacokinetic aspects of rosuvastatin. J. Clin. Pharmacol. 42, 963-970. doi: $10.1177 / 0091270002042009002$

Zweig, M. H., and Campbell, G. (1993). Receiver-operating characteristic (ROC) plots: a fundamental evaluation tool in clinical medicine. Clin. Chem. 39, 561-577.

Conflict of Interest Statement: The authors declare that the research was conducted in the absence of any commercial or financial relationships that could be construed as a potential conflict of interest.

Copyright (C) 2018 Cai, Bai, Lei, Wu, Liu, Zhu, Zhang, Liu, Lin, Chen, Zhang, He, Geng, Huang and Zhong. This is an open-access article distributed under the terms of the Creative Commons Attribution License (CC BY). The use, distribution or reproduction in other forums is permitted, provided the original author(s) and the copyright owner are credited and that the original publication in this journal is cited, in accordance with accepted academic practice. No use, distribution or reproduction is permitted which does not comply with these terms. 